\title{
Wireless Channel Characterization and System Analysis of Complex Utility Tunnel Environments ${ }^{\dagger}$
}

\author{
Mikel Celaya-Echarri 1, Leyre Azpilicueta 1,*, Peio Lopez-Iturri 2,3, Imanol Picallo 2,3, \\ Erik Aguirre ${ }^{2}$, Jose Javier Astrain ${ }^{4}$, Jesús Villadangos ${ }^{4}$ and Francisco Falcone ${ }^{2,3}$ \\ 1 School of Engineering and Sciences, Tecnologico de Monterrey, Monterrey 64849, NL, Mexico; \\ mikelcelaya@gmail.com \\ 2 Electric, Electronic and Communication Engineering Department, Public University of Navarre, \\ 31006 Pamplona, Navarra, Spain; peio.lopez@unavarra.es (P.L.-I.); imanol.picallo@unavarra.es (I.P.); \\ erik.aguirre@unavarra.es (E.A.); francisco.falcone@unavarra.es (F.F.) \\ 3 Institute of Smart Cities, Public University of Navarre, 31006 Pamplona, Navarra, Spain \\ 4 Mathematical Engineering and Computer Science Department, Public University of Navarre, \\ 31006 Pamplona, Spain; josej.astrain@unavarra.es (J.J.A.); jesusv@unavarra.es (J.V.) \\ * Correspondence: leyre.azpilicueta@tec.mx; Tel.: +52-81582082; Fax: +52-8183597211 \\ + Presented at the 6th International Electronic Conference on Sensors and Applications, \\ 15-30 November 2019; Available online: https://ecsa-6.sciforum.net/.
}

Published: 14 November 2019

\begin{abstract}
The evolution of wireless communications has led to the adoption of a wide range of applications and utilities not only by the general public but also by administrative authorities. Consequently, the huge growth of new city services requires in some specific cases the construction of underground tunnels in order to reduce visual impact within the city center, as well as enabling the maintenance and operation works of utilities. One of the main challenges is that, inherently, underground service tunnels lack coverage from exterior wireless systems, such as mobile networks or municipal WLAN networks, which can be potentially dangerous for maintenance personnel working within the tunnels. In this work, wireless channel characterization for urban tunnel scenarios will be analyzed based on the assessment of LoRaWAN and ZigBee technologies operating at $868 \mathrm{MHz}$. For this purpose, a real urban utility tunnel has been modeled and simulated by means of an in-house 3D ray launching code and compared with experimental measurements, showing good agreement. The singularity and complexity of the limited tunnel dimensions and the inclusion of additional elements such as service trays, user pathways, and handrails have been considered. Results provide an adequate radio planning approach for the deployment of wireless systems in urban utility scenarios, with optimal coverage and enhanced quality of service. Besides, in order to have access to the data obtained by the potential WSN deployed within the tunnels, a solution to store such information in a cloud is included in the study.
\end{abstract}

Keywords: wireless sensor networks; LoRaWAN; ZigBee; 3D ray launching; tunnel environment; smart cities

\section{Introduction}

The evolution of wireless communication has led to its adoption in a wide range of applications, including utilities and administrative authorities. In addition, the growth in population as well as in the services offered in cities has led in some cases to the construction of underground tunnels in order to reduce visual impact within the city center, as well as facilitating maintenance and operation works of utilities. One of the issues that local authorities have considered is the fact that, inherently, 
underground service tunnels lack coverage from exterior wireless systems, such as mobile networks or municipal WLAN networks, which can be potentially dangerous for maintenance personnel working within the tunnel. Therefore, wireless coverage should be deployed within the underground installation in order to guarantee real-time connectivity.

Radio propagation within the interior of galleries and tunnels has been studied for different wireless systems [1-3], as well as for different tunnel geometries [4]. Other approaches have developed diffraction-based models, such as the alternate direction implicit parabolic equation method [5], the boundary element method [6], numerical calculation of vector parabolic equation [7], or the application of perturbation theory. Another approach is based on the use of deterministic methods, such as ray launching (RL) or ray tracing (RT). One of the main issues is inherent divergence within the ray launching calculation as distance increases between transmit and receive antenna. In this case, solutions in scaled frequency models have been given by reconstructing the initial wave front of the source, achieved by ray density normalization [8]. One of the approaches adopted to perform wireless characterization within tunnels has been the adoption dielectric waveguide models, in which the tunnel is emulated by a waveguide with dielectric walls [9]. These models, however, do not take into account additional complexity, which appears in underground service tunnels, such as ducts, walkways, or distribution trays. Complete modeling can be achieved by employing full wave electromagnetic simulation techniques, such as Finite Difference Time Domain (FDTD) or Frequency Domain Finite Element techniques $[10,11]$. However, the computational cost can be very high, due to the large size of the tunnel structure, compared to the wavelengths considered in conventional wireless communication systems. Moreover, tunnels analyzed are usually devoted to road traffic or trains, with inherently larger cross-sections and lower structural complexity than utility tunnels, with dense obstacle distribution.

In this paper, an in-house 3D RL code is employed to analyze wireless propagation in urban tunnel scenarios at $868 \mathrm{MHz}$ operation frequency. By adequately modeling the tunnel structure, a precise characterization for the complete extension of the tunnel can be obtained.

The paper is organized as follows: the considered underground scenario to be assessed is described in Section 2. Section 3 presents the radio channel analysis and the required convergence analysis due to the particular characteristics of the scenario. In Section 4, WSN deployment issues are discussed. Finally, discussion and future works are given in Section 5.

\section{Scenario under Analysis}

In this work, the underground utility tunnels located in the Old Town of Pamplona (Navarre, Spain) have been analyzed. Figure 1a,b shows the location and a real picture of the considered tunnels. The tunnel under analysis was built in the early 2000s, under some specific streets, with the aim of easing maintenance operations. The tunnel consists of an outer layer of connected cement rings, with the inner part allocating several metallic trays for the corresponding utilities. A metallic hallway was also installed in order to provide access to maintenance personnel, as can be seen in the figure. Currently, they provide a route for electrical connections, telephone connections, waste disposal, and water distribution systems of the neighborhood.

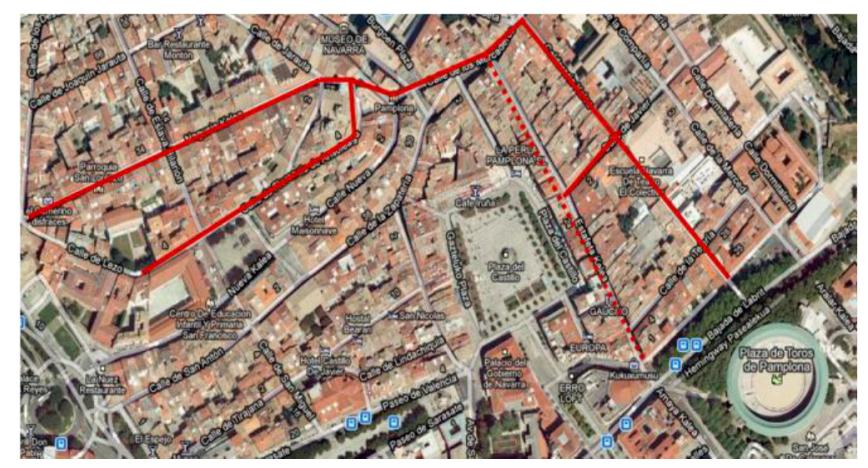

(a) 


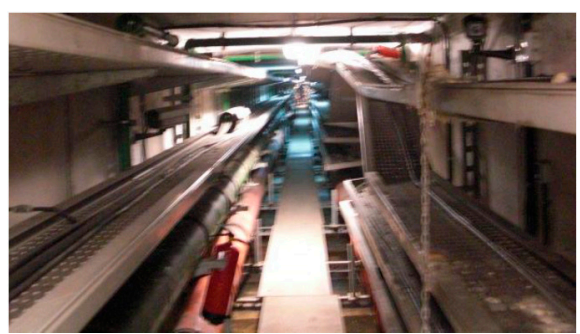

(b)

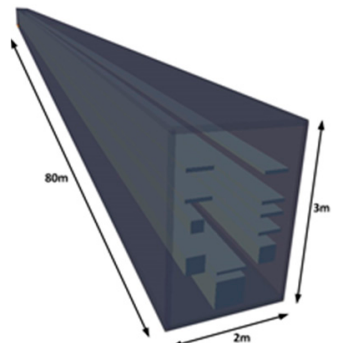

(c)

Figure 1. (a) Google Earth view of Pamplona, with the utility tunnels marked in red, (b) layout of one of the underground utility tunnels, and (c) simulation model created for the 3D ray launching (RL).

Regarding security, the utility tunnels include common intrusion detection and antifire systems, with their corresponding local alarms. One of the main concerns of Municipal Authorities is the lack of wireless coverage within the tunnels, which initially was planned to be provided by external infrastructures, such as municipal WiFi of Mobile Networks (3G/4G). However, in reality, a specific indoor coverage system is required in order to, at least, provide coverage to the maintenance staff that need to come to the tunnels.

The tunnel is not a conventional tunnel (i.e., a free hallway with walls that can be smooth or can present a certain degree of surface roughness and hence diffuse scattering), due to the fact that there is a set of service trays placed inside it. This leads to new interactions with the electromagnetic waves launched by the source, with strong multipath component generation given the large ratio between the length of the tunnel in comparison with the width of the tunnels, tray dimension, and inter-tray distance. This condition implies the need for including more precise topological information of the scenario in order to consider the same interaction, specifically for launched rays within a ray launching algorithm, as the one employed in this work.

\section{Radio Channel Analysis}

In order to characterize the radio channel within the proposed utility tunnel, simulations by means of the 3D RL in-house developed code are performed. The algorithm is based on geometrical optics (GO) and geometrical theory of diffraction (GTD). The code has been programmed in Matlab, and an exhaustive description of its operation procedure is presented in [12].

Figure 1c shows the created tunnel scenario. Real material properties have been considered: cement walls, aluminum trays, and PVC tubes of different dimensions. The dimensions of the tunnel section are as follows: width $2 \mathrm{~m}$, height $3 \mathrm{~m}$, and section length $80 \mathrm{~m}$. Large-scale propagation analysis has been performed by means of the RF power distribution for the whole volume of the scenario. For this purpose, the transmitter antenna has been placed at the beginning of the tunnel model, at a height of $2 \mathrm{~m}$ (represented by a red triangle in Figure 2). The operation frequency has been set to $868 \mathrm{MHz}$ and the transmitted power to $-10 \mathrm{dBm}$, emulating a ZigBee or LoRaWAN system at this specific frequency. Figure 2 shows the obtained RF power distribution estimations for the $X Y$ bi-dimensional plane at a height of $2 \mathrm{~m}$ (the same height as the transmitter antenna). The variability of received power along the distance can be seen, along with some spatial points with lower received power in the final part of the tunnel. 


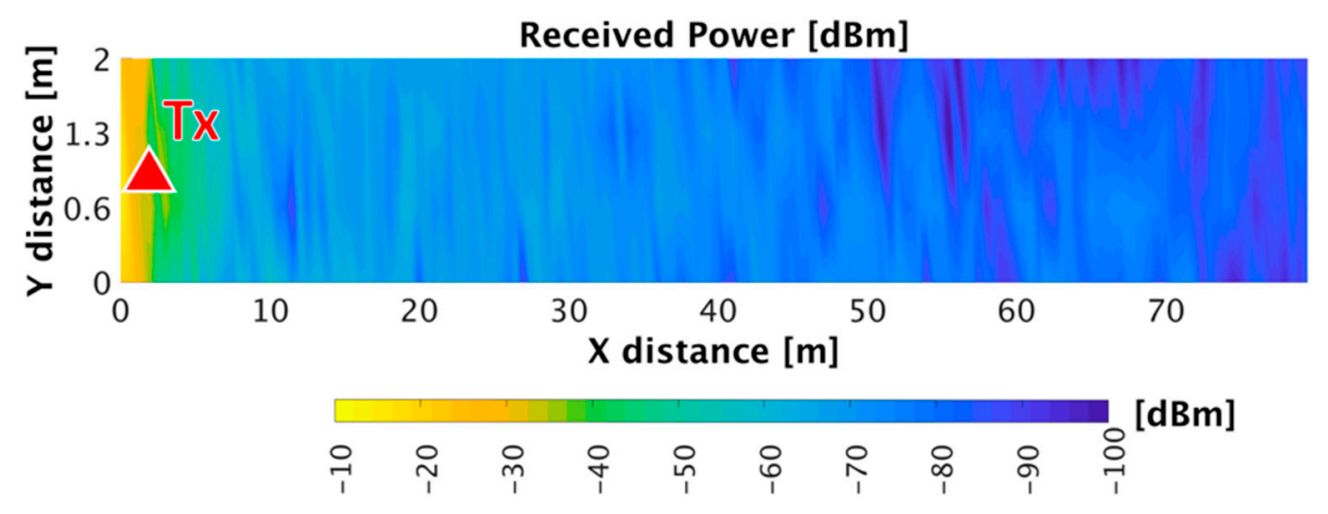

Figure 2. RF power distribution for the XY bi-dimensional plane at $2 \mathrm{~m}$ height for $868 \mathrm{MHz}$.

\section{Convergence Analysis of the Scenario}

The underground tunnel scenario has distinctive characteristics that convert it in a particular scenario for radio wave propagation purposes. These peculiar characteristics are that it is an area that is considerably long ( $80 \mathrm{~m}$ long in this specific case) and extremely narrow ( $2 \mathrm{~m}$ width). The convergence analysis of the in-house RL algorithm to obtain the optimal input parameters for best performance of the algorithm in typical indoor complex scenarios can be found in the literature [12]. However, for this specific environment, those parameters are not optimal because the RL algorithm diverges due to the extremely narrow width of the scenario. The divergence of the algorithm means that there are a lot of cuboids of the scenario that do not have any rays if the same simulation parameters are used for this specific scenario.

Accordingly, a specific convergence analysis of the algorithm has been done for this underground tunnel environment in order to characterize 3D RL convergence as a function of multiple parameters, such as cuboid dimensions, angular resolution, or maximum number of reflections. Due to the specific characteristics of the utility tunnel environment (i.e., strong reflections due to partial conductor material presence and large obstacle density), the maximum number of reflections and the angular resolution of launching rays have been assessed. Once the simulation results for the different cases have been obtained, radio channel measurements inside the tunnel section have been performed with the aim of validating those simulation results. The measurements have been carried out with the aid of an Agilent CSA N1996A to generate the RF signal and an Agilent FieldFox N9912A portable spectrum analyzer to measure the RF signal power. The transmitter has been connected to a $5 \mathrm{dBi}$ monopole antenna, whereas the receiver has been connected to small vertical monopole antenna. The considered frequency of operation has been $868 \mathrm{MHz}$ with a transmitted power of $-10 \mathrm{dBm}$. The transmitter antenna has been placed at the beginning of the tunnel, located at the center with a height of $2 \mathrm{~m}$, whereas the measurement points have been placed in a linear path in front of the transmitter, along the tunnel ( $2 \mathrm{~m}$ distance between them). Measurements have been performed with no users crossing the tunnels or other potential timevarying scatterers within the scenario. The results, compared with the different simulation cases, are depicted in Figure 3. Cases 1, 3, 4, and 5 do not have good agreement with measurements after approximately $50 \mathrm{~m}$. This is because Cases 3, 4, and 5 consider lower angular resolutions ( 1 and 0.5 degrees versus 0.2 degrees). In Case 1 , the angular resolution is high but the number of reflections is the lowest. On the other hand, Case 2 and Case 6 present a good match with real measurements. Therefore, we can conclude that the 3D RL simulation algorithm has been calibrated to be employed in this particular underground scenario. 


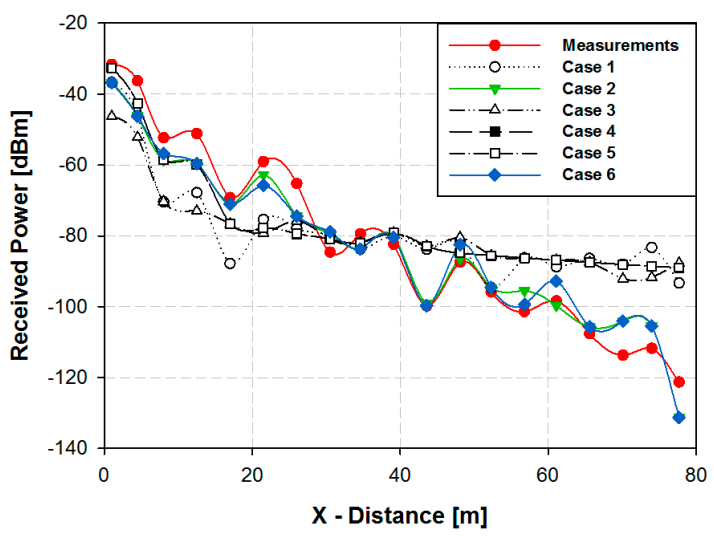

Figure 3. Comparison of different cases of simulation and measurement results for the service tunnel section under analysis.

\section{WSN Deployment}

The 3D RL simulations were shown to be accurate for an optimized radio planning analysis in this complex and particular underground scenario. Based on these preliminary results, the coverage for both Zigbee and LoRaWAN wireless devices can be estimated (i.e., the number of wireless nodes required for the whole tunnel), taking into account that ZigBee and LoRaWAN devices' sensitivity is around $-100 \mathrm{dBm}$ and $-148 \mathrm{dBm}$, respectively. Besides, the network topology is of great importance, since the tunnels' particular morphology could limit the use of star topology (the typical one for LoRaWAN).

Regarding the data collected by the WSN, it will be stored, processed, and analyzed in a private cloud. For this purpose, we have implemented an Apache CloudStack over hypervisors VMware ESXI managed by a VMware vSphere. The resulting infrastructure allows the agile and easy deployment and management of services. It allows computing orchestration, user and account management, and native APIs. Figure 4 (right) depicts the hardware infrastructure where the cloud is implemented, and the interface of the vSphere client (Figure 4, left) where different virtual machines are deployed. The cloud provides data storage, as well as data visualization with the aid of a Grafana tool, and data analytics by means of a BIRT report tool. The resulting computing cloud is easily scalable and allows hot migrations according to the availability of the servers.
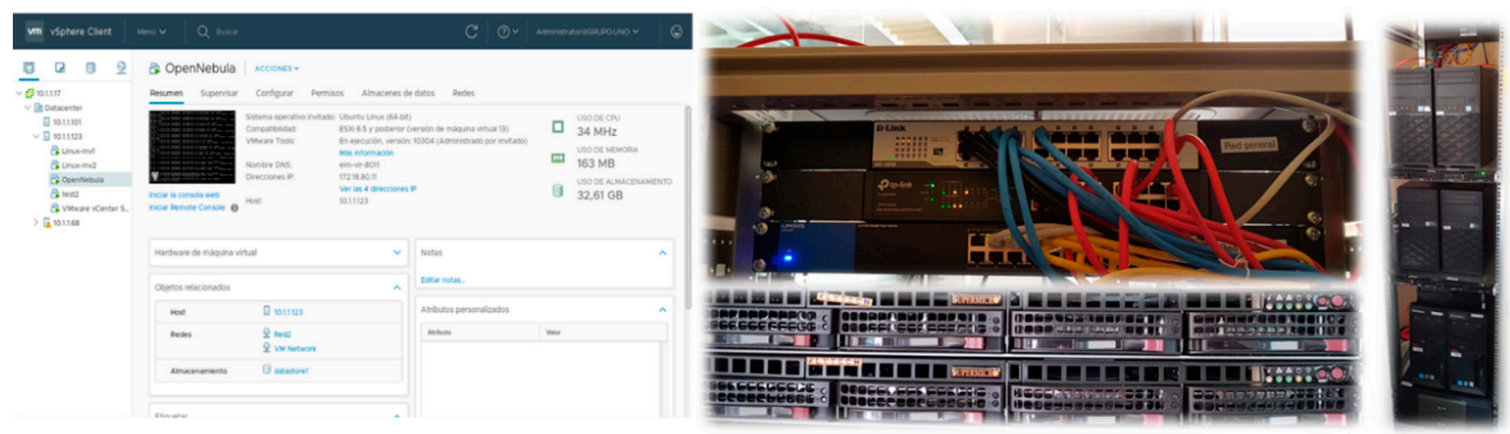

Figure 4. vSphere client (left) and hardware infrastructure (right).

\section{Discussion and Future work}

In this work, the analysis of wireless system deployment in urban utility tunnels is described. The impact of large obstacle density, given by the inclusion of elements such as service trays or handrails in the case of underground utility tunnels, is analyzed by means of in-house 3D ray launching code. Complete volumetric analysis is performed in order to consider the location of wireless transceivers and enable them to be embedded within the elements of the tunnels. Systemlevel analysis considering conventional transceiver parameters is performed, obtaining initial deployment planning results. The proposed methodology enables one to consider specific 
propagation losses in urban utility tunnels, in order to deploy wireless systems with optimal coverage, minimum interference, and enhanced quality of service. Future developments require analyzing longer tunnel runs and their impact in wireless channel analysis, in which the high density of scatterers will influence overall channel characterization, in terms of convergence analysis. Moreover, in depth characterization of wireless sensor configurations is required in order to provide an assessment of the election of systems such as ZigBee or LoRaWAN, as a function of topology, maximum transmission rate, and receiver sensitivity thresholds.

Author Contributions: Conceptualization, L.A. and P.L.-I.; methodology, L.A.; software, M.C.-E. and I.P.; validation, M.C.-E.; formal analysis, M.C.-E., L.A. and P.L.-I.; investigation, M.C.-E. and L.A.; data curation, M.C.-E. and I.P.; writing - original draft preparation, L.A. and M.C.-E.; writing-review and editing, P.L.-I. and F.F.; visualization, E.A. and M.C.-E.; supervision, F.F.; wireless network planning, P.L.-I. and I.P.; cloud configuration, J.J.A. and J.V.; funding acquisition, F.F. All authors have read and agreed to the published version of the manuscript.

Funding: This research and APC was funded by Project RTI2018-095499-B-C31, Funded by Ministerio de Ciencia, Innovación y Universidades, Gobierno de España (MCIU/AEI/FEDER, UE).

Acknowledgments: The authors would like to acknowledge the support and collaboration of the Focus Group of Telecommunications and Networks at Tecnologico de Monterrey.

Conflicts of Interest: The authors declare no conflict of interest. The statements made herein are solely the responsibility of the authors.

\section{References}

1. Delogne, P. EM Propagation in Tunnels. IEEE Trans. Antennas Propag. 1991, 39, 401-406.

2. Mariage, P.; Lienard, M.; Degauque, P. Theoretical and Experimental Approach of the Propagation of High Frequency Waves in Road Tunnels. IEEE Trans. Antennas Propag. 1994, 42, 75-81.

3. Chen, S.; Jeng, S. SBR Image Approach for Radiowave Propagation in Tunnels with and without Traffic. IEEE Trans. Antennas Propag. 1996, 45, 570-578.

4. Mahmoud, S. Wireless Transmission in Tunnels with Non-Circular Cross Section. IEEE Trans. Antennas Propag. 2010, 58, 613-616.

5. Martelly, R.; Janaswamy, R. An ADI-PE Ap proach for Modeling Radio Transmission Loss in Tunnels. IEEE Trans. Antennas Propag. 2009, 57, 1759-1770.

6. Sakai, K.; Koshiba, M. Analysis of electromagnetic field distribution in tunnels by the boundary-element method. IEE Proc. 1990, 137, 202-208.

7. Bernardi, P.; Caratelli, D.; Cicchetti, R.; Schena, V.; Testa, O. A Numerical Scheme for the Solution of the Vector Parabolic Equation Governing the Radio Wave Propagation in Straight and Curved Rectangular Tunnels. IEEE Trans. Antennas Propag. 2009, 57, 3249-3257.

8. Didascalou, D.; Schäfer, T.M.; Weinmann, F.; Wiesbeck, W. Ray-Density Normalization for Ray-Optical Wave Propagation Modeling in Arbitrarily Shaped Tunnels. IEEE Trans. Antennas Propag. 2000, 48, 13161325.

9. Zhang, Y.P.; Hwang, Y. Enhancement of rectangular tunnel waveguide model. Asia Pac. Microw. Conf. 1997, $1,197-200$.

10. Mohammed, F.H.; Samir, F.M. A High-Order Compact-FDTD Algorithm for Electrically Large Waveguide Analysis. IEEE Trans. Antennas Propag. 2008, 56, 2589-2598.

11. Rana, M.; Sanagavarapu, A. Segmented-Locally-One-Dimensional-FDTD Method for EM Propagation Inside Large Complex Tunnel Environments. IEEE Trans. Magn. 2012, 48, 223-226.

12. Azpilicueta, L.; Rawat, M.; Rawat, K.; Ghannouchi, F.; Falcone, F. Convergence Analysis in Deterministic 3D Ray Launching Radio Channel Estimation in Complex Environments. ACES J. 2014, 29, 256-271. 